\title{
A Patient with Metastatic Microsatellite Instability-High Pancreatic Ductal Adenocarcinoma with a Prolonged Response to Single- Agent Pembrolizumab
}

\author{
Annie A Guedikian Megan E Randall Anita Sharko William T Leslie
}

Section of Medical Oncology, Division of Hematology/Oncology/Cell Therapy, Department of Internal Medicine, Rush University Medical Center, Chicago, IL, USA

Keywords

Metastatic pancreatic ductal adenocarcinoma - Mismatch repair deficiency $\cdot$ Microsatellite instability · Programmed cell death protein 1 inhibitor

\section{Abstract}

Immunotherapy is an effective new approach in the treatment of many malignancies. However, pancreatic ductal adenocarcinoma (PDAC) does not usually respond to immunotherapy. We discuss the case of a patient with metastatic microsatellite instability-high PDAC who had a prolonged response to single-agent pembrolizumab for almost 3 years.

(c) 2021 The Author(s).

Published by S. Karger AG, Basel

\section{Introduction}

Metastatic pancreatic ductal adenocarcinoma (PDAC) is associated with a 5-year survival rate of $3 \%$ and a median overall survival on the order of months [1,2]. Immunotherapy continues to be explored as a potential treatment option in pancreatic tumors with microsatellite instability-high (MSI-H) or mismatch repair deficiency (dMMR). The FDA approval for the anti-PD-1 monoclonal antibody pembrolizumab stems from a pivotal phase II clinical trial that demonstrated the efficacy of PD- 1 inhibition in 12 different dMMR cancer types, with objective radiographic responses in $53 \%$ of patients and complete responses in $21 \%$ of patients [3]. 
This trial included 8 patients (9\%) with MSI-H PDAC: 2 patients had a complete response, 3 had a partial response, and 1 had stable disease, with a response rate of $62 \%$ in PDAC. Although the MSI-H/dMMR phenotype is rare in PDAC (0.8\%) [4], the American Society of Clinical Oncology practice guidelines recommend routine testing for MSI-H or dMMR and treatment with pembrolizumab as the second-line therapy for patients testing positive for MSI-H or dMMR [5]. The National Comprehensive Cancer Network guidelines also recommend MSI and/or MMR testing in patients with locally advanced or metastatic PDAC and treatment with pembrolizumab only for MSI-H or dMMR tumors [6]. Long-term outcomes of treatment remain uncertain. We present a case of a patient with metastatic PDAC and an MSI-H phenotype who had a prolonged response to single-agent pembrolizumab.

\section{Case Report/Case Presentation}

The patient is a 64-year-old female who was diagnosed with metastatic PDAC in March of 2017. She initially presented with weight loss, nausea, and abdominal pain. Imaging showed a large $7-\mathrm{cm}$ invasive cystic pancreas mass with metastases to nearby lymph nodes. A biopsy showed invasive moderately differentiated adenocarcinoma. She received 8 cycles of FOLFIRINOX from March 2017 to July 2017, and a subsequent PET/CT showed a nearcomplete response. She had developed hematological toxicities including neutropenia, anemia, and thrombocytopenia, and the treatment regimen was changed to gemcitabine and paclitaxel in December 2017.

In March 2018, a CT scan showed disease progression as evidenced by extension of the primary pancreatic head mass, retroperitoneal lymphadenopathy, and an enlarged left paratracheal lymph node. Genomic testing was performed which revealed that the tumor was MSI-H with loss of MLH1 and PMS2. She began treatment with pembrolizumab every 3 weeks starting in March 2018. Just 2 months later, CT imaging showed a decrease in the size of the primary pancreatic tumor as well as the thoracic and abdominal lymphadenopathy, consistent with a treatment response. Follow-up CT scans in May 2019 continued to show sustained response to treatment. The patient continued to receive pembrolizumab for the next 34 months. She tolerated the treatment with no significant side effects.

In January 2021, imaging demonstrated slight progression and severe narrowing of the superior mesenteric vein. The patient had received 44 cycles of pembrolizumab by this time, and pembrolizumab was discontinued. The patient then enrolled in a protocol at the University of Chicago in March 2021 using an anti-CD137 monoclonal antibody.

\section{Discussion/Conclusion}

PDAC is characterized by a low tumor mutational burden which results in poor response to immunotherapies [7]. However, a small subset of PDAC patients with dMMR have an accelerated accumulation of random mutations resulting in high levels of microsatellite instability [8]. The high mutation burden results in the synthesis of mutation-associated neoantigens presented by major histocompatibility complex class I molecules. This attracts cytotoxic $\mathrm{T}$ lymphocytes to the tumor microenvironment via T-cell receptor engagement with major histocompatibility complex [9]. However, T cells can be inactivated by binding of the PD-1/ PD-L1 complex between T cells and tumor cells. Blockade of the PD-1-PD-L1 interaction with a monoclonal anti-PD-1 antibody such as pembrolizumab results in T-cell activation and destruction of the tumor [10]. While MSI-H/dMMR is rare in PDAC, our case demonstrates that this treatment approach can be highly effective in these patients. Currently, several clinical

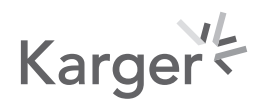


trials involving patients with MSI-H status are recruiting PDAC patients to assess the role of immunotherapy in the treatment of PDAC patients with MSI-H tumors [11].

\section{Acknowledgment}

The authors would like to acknowledge their patient who is a strong and resilient cancer survivor.

\section{Statement of Ethics}

This study was acknowledged by the Rush University Medical Center Non-Human Subject Research Committee, and ethical approval was not required. Written informed consent was obtained from the patient for publication of this case report and any accompanying images.

\section{Conflict of Interest Statement}

The authors have no conflicts of interest to declare.

\section{Funding Sources}

This study did not receive funding.

\section{Author Contributions}

Annie A. Guedikian contributed to literature review and manuscript writing. Megan E. Randall contributed to conception of the work, drafting the manuscript and revising it critically, and final approval for the version to be published. Anita Sharko contributed to conception of the work, reviewing the manuscript, and revising it critically. William T. Leslie contributed to conception of the work, drafting the manuscript and revising it critically, and final approval for the version to be published.

\section{Data Availability Statement}

All data generated or analyzed during this study are included in this article. Further enquiries can be directed to the corresponding author.

\section{References}

1 Siegel RL, Miller KD, Jemal A. Cancer statistics, 2016. CA Cancer J Clin. 2019 Jan;66(1):7-30.

2 Tas F, Sen F, Keskin S, Kilic L, Yildiz I. Prognostic factors in metastatic pancreatic cancer: older patients are associated with reduced overall survival. Mol Clin Oncol. 2013;1(4):788-92.

3 Le DT, Durham JN, Smith KN, Wang H, Bartlett BR, Aulakh LK, et al. Mismatch repair deficiency predicts response of solid tumors to PD-1 blockade. Science. 2017;357:409-13.

4 Hu ZI, Shia J, Stadler ZK, Varghese AM, Capanu M, Salo-Mullen E, et al. Evaluating mismatch repair deficiency in pancreatic adenocarcinoma: challenges and recommendations. Clin Cancer Res. 2018;24(6):1326-36.

\section{Karger's}


5 Sohal DPS, Kennedy EB, Khorana A, Copur MS, Crane CH, Garrido-Laguna I, et al. Metastatic pancreatic cancer: ASCO clinical practice guideline update. J Clin Oncol. 2018;36:2545-56.

6 Tempero MA, Malafa MP, Chiorean EG, Czito B, Scaife C, Narang AK, et al. Pancreatic adenocarcinoma, version 1.2019. J Natl Compr Canc Netw. 2019;17:202-10.

7 Yarchoan M, Hopkins A, Jaffee EM. Tumor mutational burden and response rate to PD-1 inhibition. N Engl J Med. 2017;377(25):2500-1.

8 Lee V, Murphy A, Le DT, Diaz LA Jr. Mismatch repair deficiency and response to immune checkpoint blockade. Oncologist. 2016 Oct;21(10):1200-11.

9 Eso Y, Seno H. Current status of treatment with immune checkpoint inhibitors for gastrointestinal, hepatobiliary, and pancreatic cancers. Therap Adv Gastroenterol. 2020;13:1756284820948773.

10 Dudley JC, Lin MT, Le DT, Eshleman JR. Microsatellite instability as a biomarker for PD-1 blockade. Clin Cancer Res. 2016 Feb;22(4):813-20.

11 Schizas D, Charalampakis N, Kole C, Economopoulou P, Koustas E, Gkotsis E, et al. Immunotherapy for pancreatic cancer: a 2020 update. Cancer Treat Rev. 2020 Jun;86:102016. 